\title{
The COXII Haplotypes and their Association with Productive Traits in Large White Breed Pigs
}

\author{
${ }^{1}$ Maria A. Kolosova, ${ }^{2}$ Lyubov V. Getmantseva, ${ }^{2}$ Nekruz F. Bakoev, ${ }^{2}$ Nare A. Akopyan, ${ }^{1}$ Anatoly Yu. \\ Kolosov, ${ }^{1}$ Alexander I. Klimenko, ${ }^{2}$ Olga V. Kostyunina and ${ }^{3}$ Varvara S. Shevtsova \\ ${ }^{I}$ Don State Agrarian University, Persianovski, 346493, Russia Southern Federal University, Rostov-on-Don, 344006, Russia \\ ${ }^{2}$ All-Russian Research Institute of Animal Husbandry Named after Academy Member L.K. Ernst, Dubrovitsy, Russian \\ ${ }^{3}$ Southern Federal University, Rostov-on-Don, 344006, Russia
}

\author{
Article history \\ Received: 21-02-2019 \\ Revised: 22-05-2019 \\ Accepted: 01-07-2019 \\ Corresponding Author: \\ Maria A. Kolosova \\ Don State Agrarian University, \\ Persianovski, 346493, Russia \\ Southern Federal University, \\ Rostov-on-Don, 344006, \\ Russia \\ Email:m.leonovaa@mail.ru
}

\begin{abstract}
The mitochondrial genome encodes key electron transfer chain (ETC) proteins which produce the vast majority of cellular ATP. Sequence analysis of maternal inherited mitochondrial DNA is an effective way to assess the individual characteristics of commercial breeding lines of pigs. The aims of our study were to analyze the nucleotide sequence of the mitochondrial gene COXII in Large White pigs, to search for polymorphisms and to evaluate their associations with pigs productive abilities and the backfat thickness. The studied pigs of Large White were divided in two groups. The first group (LW-1) was consisted of pigs bred in Russian Federation since 1998. The second group (LW-2) included pigs of Large White imported in 2014 to Russia from England. To evaluate the associations of the COXII gene haplotypes with productive qualities, 96 sow LW-1 were selected. The variability analysis of the COXII revealed the presence of three haplotypes: Hap_1 (TTTCTGGAT, in positions m.8292, m.8334, m.8419, m.8466, $\mathrm{m} .8526$, m.8634, m.8664, m.8682, m.8682, m.8694 respectively), Hap_2 (CCCCCAAGC) and Hap_3 (TTTTTGGAT). We have obtained the results on the association of the COXII polymorphic variants in the loci (m.C8292, m.C8334, m.C8419, m.C8526, m.A8634, m.A8664, m.G8682, m.C8694) that are typical for Hap_2, with higher value of backfat thickness in pigs.
\end{abstract}

Keywords: MtDNA, COXII, Nucleotide Sequence, Gene Position, Haplotypes, Pig, Productive Qualities, Backfat Thickness

\section{Introduction}

Mitochondria play an important role in metabolic processes as well as in apoptosis and cell aging. Polymorphism identified in mitochondrial DNA is one of the most common genetic markers used in population studies of many animal species. It's caused by the high rate of mtDNA mutations and the fact of the strict maternal fashion inheritance of mitochondria.

Both male and female individuals get mitochondria from the cytoplasm of the maternal egg. MtDNA is not able to recombination, the entire molecule is changed only by mutation for thousands of years (Fernandez et al., 2008; Nisztuk-Pacek et al., 2018). Pigs mtDNA is an annular molecule consisting of average of $16.5 \mathrm{kbp}$. In pigs it includes 37 genes: 13 for proteins of the Electron Transfer Chain (ETC), 22 for tRNA and two rRNAs (16S rRNA and 12S rRNA), as well as the most variable region, the D-loop (Yen et al., 2007).
For billions of years, the mitochondrial genome has evolved as organisms have adapted to the environments (Srirattana et al., 2017). As a result, mutations have become fixed. Various mitochondrial lineages have arisen from one or several common sources - mitochondrial genomes, which are characterized by clustering into groups known as mtDNA haplotypes (Wallace et al., 2003). Consequently, mtDNA haplotypes cause differences between breeds and are associated with both advantages and disadvantages to the organism (Wallace et al., 2003).

In cattle associations with calving (Sutarno et al., 2002), meat quality (Mannen et al., 2003), milk (Bell et al., 1985), embryo production efficiency (Tamassia et al., 2004; Bruggerhoff et al., 2002) and body weight (Derr et al., 2012) were identified. In pigs associations with body weight (Yen et al., 2007) and meat quality (Fernandez et al., 2008) was discovered. Furhtemore, the associations with the reproductive capacity and litter size in 
pigs have been reported (Tsai et al., 2016; Justin et al., 2018) and productive and reproductive traits in Afec-Assaf sheep (Reicher et al., 2012).

The vast majority of the mitochondrial genome mutations belongs to the class 1 (silent mutations (third codon)), while the mutations of class 2 (first and second positions of codons) lead to an amino acid substitution. In the genes that encode the mitochondrial proteins the observed frequency of substitutions is much higher for the sites of class 1 than for the sites of class 2 . This is true for the gene of subunit II of cytochrome oxidase $\mathrm{C}$ (COXII gene). The sites of class 1 accumulate substitutions faster than sites of class 2 .

The mtDNA gene COXII is one of the electron transfer chain complex IV elements. Due to this fact it plays a key role in energy production. Therefore the mutations in the COXII gene can be associated with economically important characteristics of pigs. Taking into account the role of the gene and the relatively high degree of intraspecies variability, the identification of polymorphisms in pigs mtDNA and the nucleotide sequence investigation of the COXII gene are very prospective (Liu and Lizhi, 2017; Nisztuk-Pacek et al., 2018).

The aim of this study was the investigation of the mtDNA COXII gene nucleotide sequence in Large White pigs, the identification of polymorphisms and the evaluation of their associations with the productive qualities of pigs.

\section{Materials and Methods}

To determine the mitochondrial COXII gene nucleotide sequence samples were collected from two groups of Large White pigs. The first group consisted of Large White pigs (LW-1) bred in Russian Federation since 1998. The second group (LW-2) included pigs of a Large White imported from England to Russia in 2014. The mtDNA was isolated from 48 tissue samples of LW-1 $(\mathrm{n}=24)$ and LW-2 $(\mathrm{n}=24))$ groups using the "K-Sorb-100" kit (Syntol, Russia) according to the manufacturer's instructions.

The sequence NC_000845.1 (Sus scrofa) published in the NCBI has chosen as reference. The COXII was amplified using forward (5'CACGGATGTCCTCCTCCCTA-3') and reverse (5'TTCTGGGCTTGCTGGGTATG-3') primers. The $25 \mu \mathrm{l}$ reaction mixture consisted of $3 \mu \mathrm{l}$ of DNA sample $(75 \mathrm{ng} / \mu \mathrm{l}), 5 \mu \mathrm{l}$ of $10 \times$ PCR-standard reaction buffer, $1 \mu \mathrm{L}$ dNTP $(1 \mathrm{mmol} / \mathrm{l}), 0.5 \mu \mathrm{l}$ of each primer $(20 \mu \mathrm{mol} / \mathrm{l})$ and 0.5 $\mu \mathrm{L}$ Taq DNA polymerase. The amplification conditions were as follows $94^{\circ} \mathrm{C}$ for $3 \mathrm{~min}$ followed by 33 cycles at $94^{\circ} \mathrm{C}$ for $30 \mathrm{~s}, 58^{\circ} \mathrm{C}$ for $30 \mathrm{~s}$ and $72^{\circ} \mathrm{C}$ for $1.5 \mathrm{~min}$. The final step was $72^{\circ} \mathrm{C}$ for $5 \mathrm{~min}$.

Specific PCR fragments were isolated from the gel using the Cleanup Mini kit for the DNA purification from the gel (Evrogen, Russia). Amplicons were sequenced using a Terminator Cycle Sequencing kit (Applied Biosystem, Foster City, CA) in the Gene Amp PCR system 9700 (Applied Biosystem) BioEdit. For editing and sequences alignment BioEdit v7.2.6 and MEGA 7 software were used.

To evaluate the associations of the COXII gene haplotypes with productive qualities, 96 sows LW-1 were selected. Productive qualities were assessed by Backfat thickness (BF) and Average Daily Gain (ADG), measured when the animals weight reached $100 \mathrm{~kg}$. Analysis of the COXII polymorphism effect on the sows productive characteristics was performed using LMM (Linear Mixed Models) (Getmantseva et al., 2017; Harrison et al., 2018). The parameters of the mixed linear model were calculated using the lme4 package of the $\mathrm{R}$ language in the $\mathrm{R}$-studio environment.

\section{Results}

At the first stage, 48 nucleotide sequences of the COXII gene were studied, 24 of the LW-1 group and 24 of the LW-2 group. As a result the nucleotide sequence of the COXII gene was determined. When analyzing the obtained data, it can be noted that the position of the COXII gene is within the interval of $8203 . .8890 \mathrm{bp}$, the size of the gene is $687 \mathrm{bp}$, which corresponds to the reference population. The studied sample includes three haplotypes, which formed by 9 substitutions in the positions $\mathrm{m} .8292, \mathrm{~m} .8334, \mathrm{~m} .8419, \mathrm{~m} .8466$, m.8526, m.8634, m.8664, m.8682, m.8682, m.8694 (Table 1).

Two haplotypes (Hap_1 and Hap_2) were identified in the LW-1 pigs. All the animals from the LW-2 group had only Hap_3. It is interesting to note that Hap_1 which have been defined for LW-1 differs from Hap_3 by only one substitution in the m.8466 locus. At the same time, Hap_2, belonging to LW-1, also differs from Hap_3 by substitution in the m.8466 locus and by substitutions in 8 other loci. Intra-group differences in LW-1, presented by Hap_1 and Hap_2, are due to substitutions in 8 loci: m.T8292C, m.T8334C, m.T8419C, m.T8526C, m.G8634A, m.G8664A, m.A8682G, m.T8694C, respectively.

To evaluate the associations of the COXII gene haplotypes with productive traits, the LW-1 sample was increased by 96 pigs. Figures 1 and 2 shows the distribution of the $\mathrm{BF}$ and $\mathrm{ADG}$ values of different haplotypes in LW-1.

Figure 1 shows that BF in pigs with Hap_1 is distributed symmetrically and has greater variability than Hap_2. It should be noted that there is a very low variety of high BF values in Hap_2. Comparison of Hap_1 and Hap_2 graphs allows us to suppose significant differences between them in the value of BF. 
Maria A. Kolosova et al. / OnLine Journal of Biological Sciences 2019, 19 (3): 146.151 DOI: 10.3844/ojbsci.2019.146.151

Table 1: Haplotypes of the COXII in large white pigs

\begin{tabular}{llllllllll}
\hline Haplotype & $\mathrm{m} .8292$ & $\mathrm{~m} .8334$ & $\mathrm{~m} .8419$ & $\mathrm{~m} .8466$ & $\mathrm{~m} .8526$ & $\mathrm{~m} .8634$ & $\mathrm{~m} .8664$ & $\mathrm{~m} .8682$ & $\mathrm{~m} .8694$ \\
\hline Hap_1 & $\mathrm{T}$ & $\mathrm{T}$ & $\mathrm{T}$ & $\mathrm{C}$ & $\mathrm{T}$ & $\mathrm{G}$ & $\mathrm{G}$ & $\mathrm{A}$ & $\mathrm{T}$ \\
Hap_2 & $\mathrm{C}$ & $\mathrm{C}$ & $\mathrm{C}$ & $\mathrm{C}$ & $\mathrm{C}$ & $\mathrm{A}$ & $\mathrm{A}$ & $\mathrm{G}$ & $\mathrm{C}$ \\
Hap_3 & $\mathrm{T}$ & $\mathrm{T}$ & $\mathrm{T}$ & $\mathrm{T}$ & $\mathrm{T}$ & $\mathrm{G}$ & $\mathrm{G}$ & $\mathrm{A}$ & $\mathrm{T}$ \\
\hline
\end{tabular}

Table 2: Differences in BF and ADG in pigs of LW-1 with Hap_1 and Hap_2 of COXII Hap of the COXII

\begin{tabular}{llllll} 
Trait & Hap_1 & Hap_2 & df & $\chi^{2}$ & p \\
\hline BF & $20.86 \pm 1.02$ & $24.01 \pm 1.58$ & 1 & 4.896 & $0.026^{*}$ \\
ADG & $783.02 \pm 22.43$ & $764.02 \pm 27.68$ & 1 & 0.586 & 0.443 \\
\hline
\end{tabular}

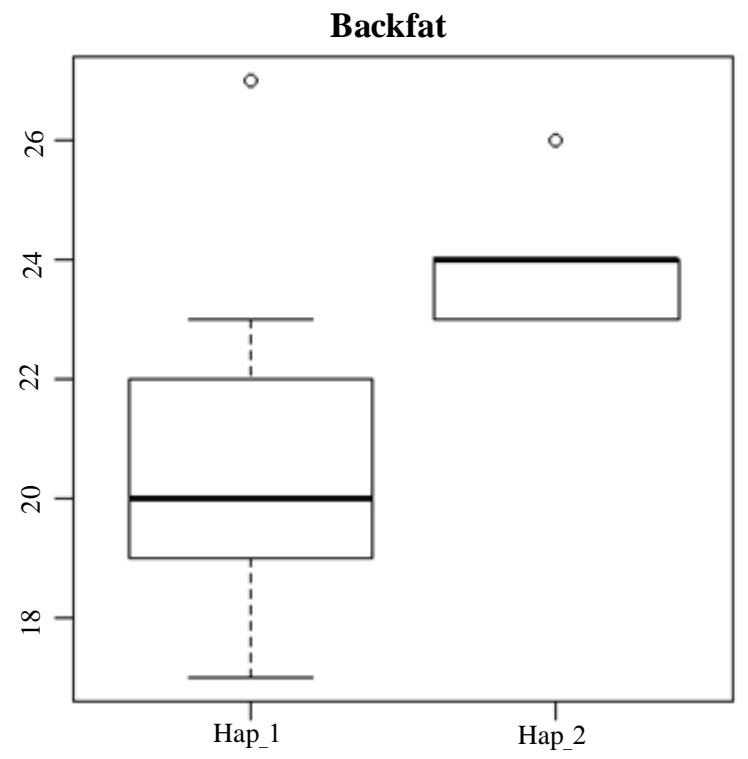

Fig. 1: Backfat thickness in pigs of the COXII Hap_1 and Hap_2

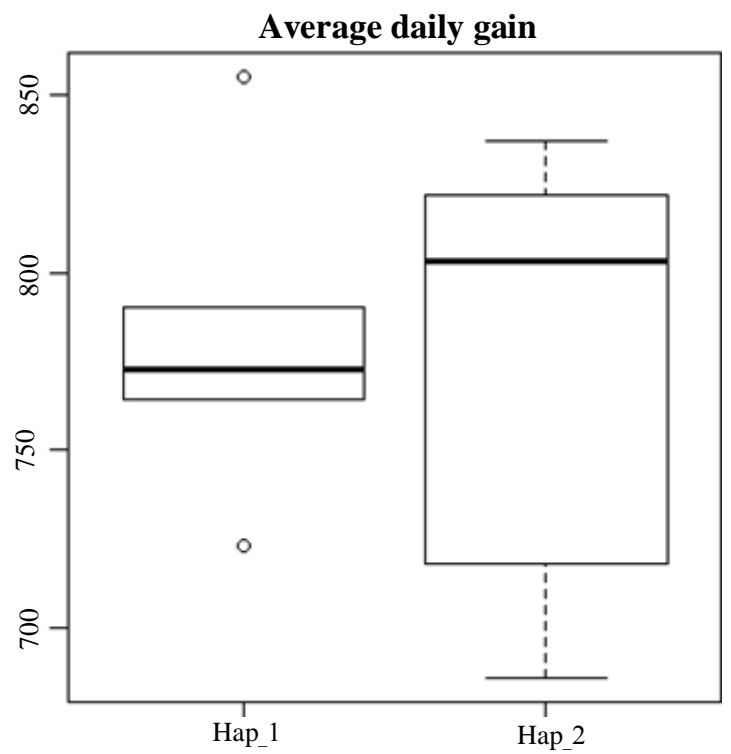

Fig. 2: Average daily gain in pigs of the COXII Hap_1 and Hap_2 
According to the graphs shown in Fig. 2, the Hap_2 group shows a high variability in the ADG compared to Hap_1.

The reliability of differences between the $\mathrm{Y}$ traits (BF and ADG) which are associated with the COXII haplotypes was assessed using Linear Mixed Model:

$$
Y i j k=\mu+H a p i+Y e a r j+P k+e i j k
$$

The fixed factors were Hap (Hap_1 and Hap_2 of the $C O X I I)$ and the Year of the animal. A randomized factor was the sire's number ( $\mathrm{P}$ - parent). As a result the following full_model on the syntax R was used:

$$
Y \sim \text { Hap }+ \text { Year }+(1 \mid P)
$$

To evaluate the conditionality of BF and ADG by factor Hap we compared full_model to null_model:

$$
Y \sim 1+\text { Year }+(1 \mid P)
$$

The obtained results have shown the significant effect of COXII haplotypes on backfat thickness (Table 2). Hap_1 was associated with a lower BF. The differencies in ADG caused by factor Hap of the COXII in pigs of LW-1 group didn't reveal.

\section{Discussion}

Studies of the polymorphism of nuclear and mtDNA allow us to uncover the unique biological characteristics of pigs and develop selection methods using molecular genetic information. Today, data on mtDNA of the main breeds are available, but the search for associations of mtDNA polymorphism with productive qualities of pigs is of increasing interest.

At present, the study of the complete mitochondrial genome of pigs is given much attention (Tsai et al., 2016; Nguyen et al., 2016; Justin et al., 2018; Kumar et al., 2019; Yang et al., 2019). The results of Tsai et al. (2016), which identified five haplotypes of mtDNA in commercial lines of Australian pigs. Their studies allowed to determine the genetic diversity of domestic pigs and to show how mtDNA haplotypes influenced Litter size, as well as ovulation rate, uterine efficiency and embryo survival. Studies by Justin et al. (2018) also demonstrated the effect of mtDNA haplotypes on fat density, muscle depth, fat-to-weight ratio, daily lifetime increase and nipple quality in pigs. However, few studies have been carried out on individual mtDNA genes and their relationship to pig traits. Due to the fact that COXII plays a key role in energy production, it is an interesting object for research.

The results indicating the relationship between the polymorphism of the COXII gene were obtained in the study of other species. (Nisztuk-Pacek et al., 2018) have obtained results, which proved the associative connections between the COXII gene polymorphisms and phenotypic characteristics of animals. They pointed to the statistically significant relationship of the mtDNA gene COXII haplotypes and haplogroups with the productive traits of raccoon dogs, such as weight, body size and colour type. Jeon et al. (2005) showed significant differences between genotypes identified in the mitochondrial gene COXII and weight in Hanwoo cattle. Results from the Ślaska et al. (2016) indicate that the COXII mitochondrial gene may be involved in hair development. The results we have obtained reflect the association of the COXII gene polymorphisms with the backfat thickness in pigs.

According to our data, these are the first studies aimed at finding significant associations between the polymorphism of the mitochondrial gene COXII and the productive qualities of pigs. We determined that Hap_1 in pigs LW-1 is associated with a lower backfat thickness. It is interesting to note that in the LW_2 group, only Hap_3 is identified. Meanwhile the backfat thickness in LW-2 $(\mathrm{BF}=14.5 \pm 0.65)$ is significantly less comparing to LW-1 $(B F=22.2 \pm 0.75)$. The reason of the difference between Hap_1 and Hap_3 is the substitution in a position m.C8466T. Also it may indicate that COXII polymorphic variants (in the positions m.C8292, m.C8334, m.C8419, m.C8526, m.A8634, m loci. A8664, m.G8682, m.C8694), which are typical for Hap_2, are associated with higher backfat thickness in pigs.

The data obtained will complement the knowledge of the molecular genetic mechanisms of the formation of productive features of pigs and can be used in the development of technology for creating breeding resources in pig breeding based on molecular genetic information. In general, the results presented by a number of scientists reveal that mtDNA haplotypes and haplogroups, including the D-loop sequence, COXI, COXII genes, tRNA and others, are associated with the productive traits of animals. Further studies in this field will assess the value of mtDNA variants as animal productive indicators as well as their using with nuclear polymorphisms for improvement of agricultural animals.

\section{Conclusion}

The variability analysis of the mtDNA COXII gene revealed the presence of three haplotypes in large white breed pigs: Hap_1 (TTTCTGGAT, in positions m.8292, m.8334, m.8419, m.8466, m.8526, m.8634, $\mathrm{m} .8664$, m.8682, m.8682, m.8694, respectively), Hap_2 (C CCCCAAGC) and Hap_3 (TTTTTGGAT). The results on the association of the COXII gene polymorphisms in loci m.C8292, m.C8334, m.C8419, m.C8526, m.A8634, m.A8664, m.G8682, m.C8694, which are typical for Hap_2, with higher backfat thickness in pigs have been obtained. 


\section{Funding Information}

The work was performed using the grant of the President of Russian Federation for supporting of young Russian scientists-candidates of sciences (contract No. MK-1443.2018.11).

\section{Acknowledgement}

We thank A.V. Usatov, Doctor of Biological Sciences, Head of the Molecular Genetics Laboratory, Southern Federal University, Russia, for valuable and constructive suggestions during the planning and development of this research work.

\section{Author's Contributions}

Maria A. Kolosova, Lyubov V. Getmantceva: Designed and performed experiments and wrote the paper.

Necruz F. Bakoev, Anatoly Yu. Kolosov, Nare A. Akopyan and Varvara S. Shevtsova: Developed analytical tools and analyzed data.

Alexandr I. Klimenko, Olga V. Kostyunina: Collected and analyzed data.

\section{Ethics}

This article is original and contains unpublished materials. The corresponding author confirms that all of the other authors have read and approved the manuscript and no ethical issues involved.

\section{References}

Bell, B.R., B.T. McDaniel and O.W. Robison, 1985. Effects of cytoplasmic inheritance on production traits of dairy cattle. J. Dairy Sci., 68: 2038-51. DOI: 10.3168 /jds.S0022-0302 (85) 81066-3

Bruggerhoff, K., V. Zakhartchenko, H. Wenigerkind, H.D. Reichenbach and K. Prelle et al., 2002. Bovine somatic cell nuclear transfer using recipient oocytes recovered by ovum pick-up: Effect of maternal lineage of oocyte donors. Biol. Reprod., 66: 367-73. DOI: 10.1095/biolreprod66.2.367

Derr, J.N., P.W. Hedrick, N.D. Halbert, L. Plough and L.K. Dobson et al., 2012. Phenotypic effects of cattle mitochondrial DNA in American bison. Conserv. Biol., 26: 1130-6.

DOI: $10.1111 /$ j.1523-1739.2012.01905.x

Fernandez, A.I., E. Alves, A. Fernandez, E. de Pedro and M.A. Lopez-Garcia et al., 2008. Mitochondrial genome polymorphisms associated with longissimus muscle composition in Iberian pigs. J. Anim. Sci., 86: 1283-90. DOI: 10.2527/jas.2007-0568
Getmantseva, L., A. Kolosov, M. Leonova, A. Klimenko and S. Bakoev et al., 2017. Effect of polymorphisms in intron 1 of the swine POU1F1 gene on growth and reproductive traits. Turkish J. Vet. Anim. Sci., 41: 643-647. DOI: 10.3906/vet-1702-77

Harrison, X.A., L. Donaldson, M.E. Correa-Cano, J. Evans and D.N. Fisher et al., 2018. A brief introduction to mixed effects modelling and multimodel inference in ecology. PeerJ. Preprints, 6: 3113-2 DOI: $10.7717 /$ peerj.4794

Jeon, G.J., H.Y. Chung, J.G. Choi, M.S. Lee and C.W. Lee et al., 2005. Relationship between genetic variants of mitochondrial DNA and growth traits in Hanwoo cattle. Asian-Australasian J. Anim. Sci., 18: 301-307. DOI: 10.5713/ajas.2005.301

Justin, C., S.t. John and T. Te-Sha., 2018. The association of mitochondrial DNA haplotypes and phenotypic traits in pigs. BMC Genetics, 19: 41 DOI: 10.1186/s12863-018-0629-4

Kumar, J.K., S.A. Pratap, A.B. Srivastav and B.C. Sarkhel, 2019. Molecular characterization of the complete mitochondrial genome sequence of Indian wild pig (Sus scrofa cristatus). Anim. Biotechnol., 30: 186-191.

DOI: $10.1080 / 10495398.2018 .1469506$

Liu, G. and Z. Lizhi, 2017. Population genetic structure and molecular diversity of the red swamp crayfish in China based on Mtdna COI gene sequences, Mitochondrial DNA Part A, 28: 860-866. DOI: $10.1080 / 24701394.2016 .1199022$

Mannen, H., M.L. Morimoto, K. Oyamat, F. Mukai and S. Tsuji, 2003. Identification of mitochondrial DNA substitutions related to meat quality in Japanese black cattle. J. Anim. Sci., 81: 68-73.

DOI: $10.2527 / 2003.81168 x$

Nguyen, H.D., P.T. Nguyen, T.A. Bui and O.T. Kim, 2016. The complete mitochondrial genome sequence of the indigenous I pig (Sus scrofa) in Vietnam. Asian Aus. J. Anim. Sci. DOI: 10.5713/ajas.16.0608

Nisztuk-Pacek, S., B. Slaska, G. Zieba and I. Rozempolska-Rucinska, 2018. Two Mitochondrial Genes Are Associated with Performance Traits in Farmed Raccoon Dogs (Nyctereutes procyonoides) Czech J. Anim. Sci., 63: 110-118. DOI: 10.17221/2/2017-CJAS

Reicher, S., E. Seroussi, J.I. Weller, A. Rosov and E. Gootwine, 2012. Ovine mitochondrial DNA sequence variation and its association with production and reproduction traits within an AfecAssaf flock. J. Anim. Sci., 90: 2084-91. DOI: $10.2527 /$ jas.2011-4673.

Ślaska, B., G. Zięba, I. Rozempolska-Rucińska, G. Jeżewska-Witkowska and S.N. Horecka et al., 2016. Mitochondrial DNA haplotypes are associated with performance traits in Raccoon dogs. Anim. Sci. Papers Reports, 34: 293-302. 
Srirattana, K., K. McCosker, T. Schatz and J.C. St John, 2017. Cattle phenotypes can disguise their maternal ancestry. BMC Genetics, 18: 59.

DOI: $10.1186 / \mathrm{s} 12863-017-0523-5$

Sutarno, C.J.M., J. Greeff and A.J. Lymbery, 2002. Mitochondrial DNA polymorphisms and fertility in beef cattle. Theriogenology, 57: 1603-10.

DOI: 10.1016/S0093-691X(02)00664-7

Tamassia, M., F. Nuttinck, P. May-Panloup, P. Reynier and Y. Heyman et al., 2004. In vitro embryo production efficiency in cattle and its association with oocyte adenosine triphosphate content, quantity of mitochondrial DNA and mitochondrial DNA haplogroup. Biol. Reprod., 71: 697-704.

DOI: 10.1095/biolreprod.103.026104
Tsai, T.S., S. Rajasekar and J.C. St John, 2016. The relationship between mitochondrial DNA haplotype and the reproductive capacity of domestic pigs (Sus scrofa domesticus). BMC Genet. 17: 67. DOI: $10.1186 / \mathrm{s} 12863-016-0375-4$

Wallace, D.C., E. Ruiz-Pesini and D. Mishmar, 2003. mtDNA variation, climatic adaptation, degenerative diseases and longevity. Cold Spring Harb Symp Quant Biol., 68:479-86. DOI: 10.1101/sq.2003.68.471

Yang, J., J. Wu, W. Ding, J. Chen and J. Xing 2019. The complete mitochondrial genome of the Fengjing Mitochondrial DNA Part B, 4: 443-445. DOI:10.1080/23802359.2018.1555014 pig

Yen, N.T., C.S. Lin, C.C. Ju, S.C. Wang and M.C. Huang 2007. Mitochondrial DNA polymorphism and determination of effects on reproductive trait in pigs. Reprod. Domest. Anim., 42: 387-92. DOI: $10.1111 / \mathrm{j} .1439-0531.2006 .00797 . x_{-}$ 